\title{
Practical Considerations Concerning HL-to -RT Equivalence Checking
}

\author{
Carl Pixley
}

Synopsys

\begin{abstract}
We will discuss several years' experience with commercial HL-toRTL equivalence checking with the Hector technology. We will also discuss several considerations based upon the reality that our company is an EDA vendor. This is quite different from the position of a semiconductor company, which can concentrate on a very specific methodology and design type.

Our observations will include some case studies from customers about the methodology and designs on which they are using Hector. Most of the development of Hector was based upon solutions to problems presented by our customers. We will also discuss the general architecture of Hector and some technological information about the engines that underlie Hector.
\end{abstract}

